\title{
Single-crystal total scattering meets metal-organic frameworks: deciphering the elusive structure of ZIF-90
}

\author{
S. Canossa ${ }^{1}$, R. Frison², X. $\mathrm{Pei}^{3}$, H.-B. Bürgi ${ }^{4}$ \\ ${ }^{I}$ EMAT, Dept. of Physics, University of Antwerp, 2020 Antwerp, Belgium, \\ ${ }^{2}$ Dept. of Physics, University of Zurich, 8093 Zurich, Switzerland, \\ ${ }^{3}$ College of Chemistry, University of California Berkeley, Berkeley, CA 9472, U.S.A. \\ ${ }^{4}$ Department of Chemistry and Biochemistry, University of Bern, CH-3012 Bern, Switzerland
}

stefano.canossa@uantwerpen.be

Modern materials science is increasingly concerned with the engineering of the periodic and aperiodic features of crystalline materials, thus making structural information on both aspects a key analytical target. In this regard, diffuse scattering from single-crystal diffraction data contains a wealth of structural information, which has been already used to unravel the local 3D structures of several inorganic and organic materials. ${ }^{[1]}$ Yet, the use of this analysis remains a long-lasting challenge in the field of one of the most researched and ubiquitous materials, metal-organic frameworks (MOFs).

In our work, we elucidated the defect structure of the renowned MOF ZIF- $90^{[2]}$ by Monte Carlo based single-crystal diffuse scattering simulations (Figure 1). Our analysis showed how correlated disorder of framework components induces lattice distortions affecting the framework local symmetry and porosity. Moreover, we observed that these characteristics are influenced by the synthetic conditions and are always present in ZIF-90 crystals. While allowing a new understanding of the structure-property relationship of this MOF, our study provides a blueprint for future total-scattering studies of MOF single crystals featuring entangled substitutional and displacive disorder.

[1] T. R. Welberry, T. Weber, Crystallography Reviews 2016, 22, 2-78.

[2] W. Morris, C. J. Doonan, H. Furukawa, R. Banerjee, O. M. Yaghi, Journal of the American Chemical Society 2008, $130,12626-12627$.
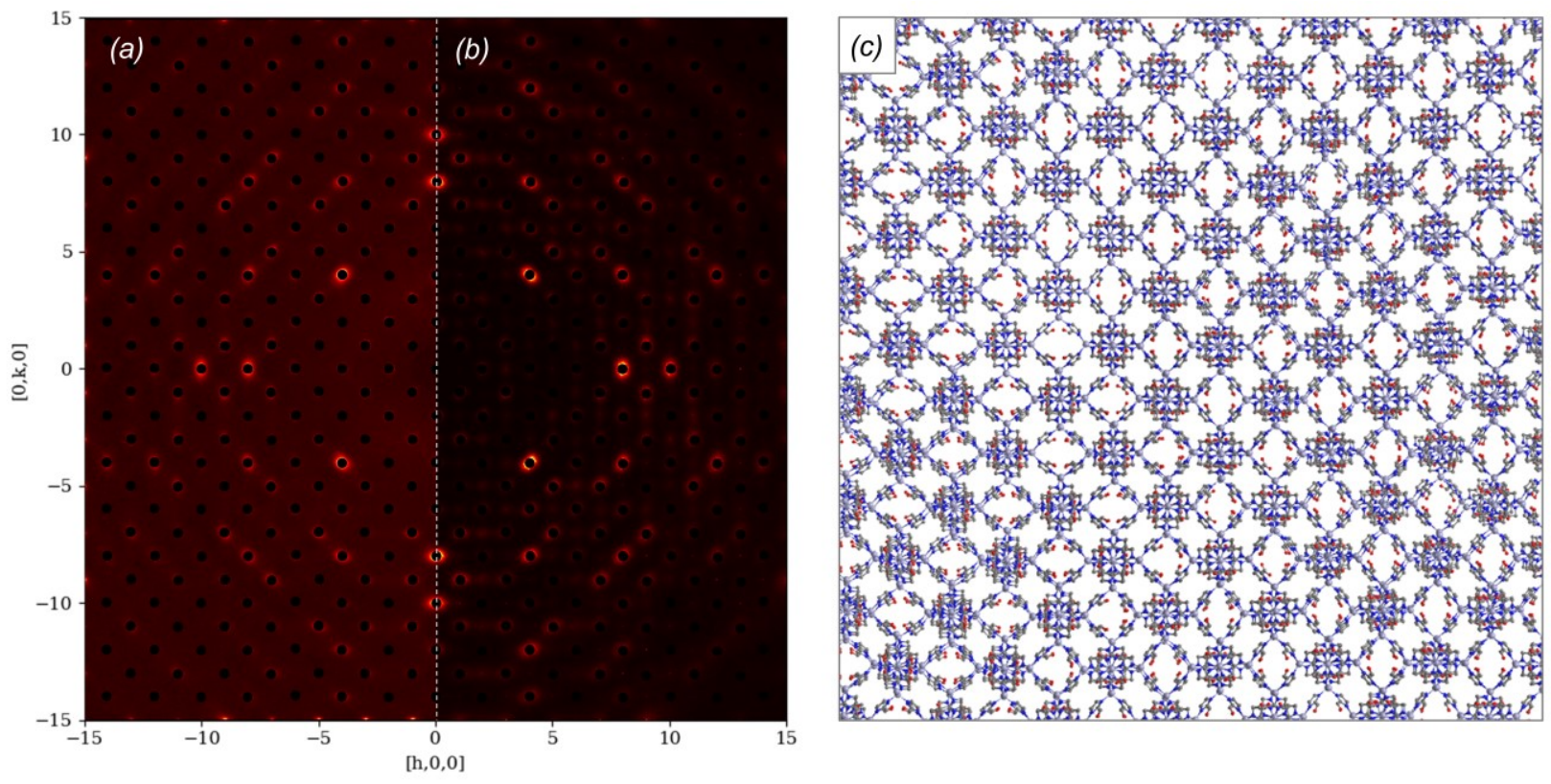

Figure 1. Experimental (a) and simulated (b) patterns of ZIF-90 with masked Bragg reflections, and partial view of the model used to reproduce the observed diffuse scattering (c).

Keywords: total scattering; correlated disorder; metal-organic frameworks; ZIF-90 